\title{
Amadeus
}

International Multidisciplinary Journal IISSN 2525-8281

DOI: 10.14295/aimj.v4i7.86

\section{Food-Dependent Exercise Anaphilaxy: A Systematic Review of Literature}

Antonio Adriano Gomes dos Santos Filho ${ }^{1}$ Modesto Leite Rolim Neto ${ }^{1}$

\begin{abstract}
Over and over again food-induced exercise-induced anaphylaxis is underdiagnosed by the physician and is reported to be an idiopathic anaphylaxis. In this context, the patient does not receive the proper guidelines for the correct management of his disease. Therefore, you will not know how to act, either to avoid it or to intervene in a possible crisis, so you will be potentially subject to life-threatening situations. Thus the research question was thus outlined: "How should the patient with fooddependent exercise-induced anaphylaxis (AIEDA) act in the face of a likely crisis, particularly in reducing the number of fatal events?" A literature review was performed. on the following bases: Online Medical Literature Search and Analysis System (MEDLINE), Scientific Electronic Library Online (SCIELO). The following controlled descriptors were selected for the location of the studies: "Wheat-Dependent Exercise-Induced Anaphylaxis", "Impending Anaphylactic Crisis", "Patients Exposed to Imminent Anaphylactic Crisis" (patients exposed to impending anaphylactic crisis). ). Exercise-induced anaphylaxis (EIA) is believed to be associated with 5-15\% of all anaphylaxis cases. AIEDA prevalence data are limited and not reported accurately. Most reported cases have been related to wheat. Reported prevalence ranged from $0.001 \%$ to $0.017 \%$ worldwide for all foods. In most patients, when there is a mediated IgE reaction, exercise decreases the threshold for mast cell degranulation, giving rise to symptoms even when isolated ingestion is tolerated. Severe food allergy sometimes develops during exercise in association with a specific food (up to four hours after ingestion), characterizing fooddependent exercise-induced anaphylaxis. Proper professional diagnosis and management will help to avoid unnecessary limitations on the patient's diet and exercise. In any situation of anaphylaxis, it is never an exaggeration to stress the importance of educating patients to know how and when to use the self-injecting adrenaline kit, and to convey the notion of ineffective premedication with antihistamines. even mask prodromal symptoms, preventing patients from predicting more severe reactions.
\end{abstract}

Keywords: Wheat-Dependent Exercise-Induced Anaphylaxis; Impending anaphylactic crisis; Patients exposed to impending anaphylactic crisis

\footnotetext{
${ }^{1}$ Juazeiro do Norte Medical Estácio School (FMJ), Juazeiro do Norte, Ceará, Brazil. Corresponding author: modestorolim@yahoo.com.br
} 


\title{
Anafilaxia Induzida por Exercício Dependente de Alimentos: Uma Revisão Sistemática da Literatura
}

\begin{abstract}
Resumo: Por inúmeras vezes a anafilaxia induzida pelo exercício dependente de alimento é subdiagnosticada pelo médico e acaba sendo relatada como uma anafilaxia idiopática. Neste contexto, o paciente não recebe as devidas orientações para o correto manejo de sua doença. Sendo assim, não saberá como agir, seja para evita-la ou para intervir em uma possível crise, logo estará potencialmente sujeito à situações de risco de vida. Desse modo a pergunta de pesquisa ficou assim delineada: "Como o paciente com anafilaxia induzida pelo exercício dependente de alimento (AIEDA) deve agir diante de uma provável crise, particularmente, na redução do número de eventos fatais?" Foi efetuada uma revisão da literatura nas seguintes bases: Sistema Online de Busca e Análise de Literatura Médica (MEDLINE), Scientific Eletronic Library Online (SCIELO). Para a localização dos estudos foram selecionados os seguintes descritores controlados: "Anafilaxia induzida pelo exercício físico" (Wheat-Dependent Exercise-Induced Anaphylaxis), "Crise anafilática iminente" (Impending anaphylactic crisis), "pacientes expostos a crise anafilática iminente" (patients exposed to impending anaphylactic crisis). ). Acredita-se que a anafilaxia induzida pelo exercício (AIE) esteja associada a $5-15 \%$ de todos os casos de anafilaxia. Os dados de prevalência da AIEDA são limitados e não relatados com exatidão. A maioria dos casos relatados tem sido relacionada ao trigo. A prevalência relatada variou de $0,001 \%$ a $0,017 \%$ em todo o mundo para todos os alimentos. Na maioria dos doentes, quando há uma reação IgE mediada, verifica-se que o exercício diminui o limiar para a degranulação dos mastócitos, dando origem aos sintomas, mesmo quando a ingestão isolada é tolerada. Algumas vezes, o quadro de alergia alimentares graves se desenvolve durante o exercício em associação com um alimento específico (até quatro horas após a ingestão), caracteriza a anafilaxia induzida pelo exercício dependente de alimento. O diagnóstico e o manejo apropriados pelo profissional ajudará a evitar limitações desnecessárias na dieta e no exercício do paciente. Perante qualquer situação de anafilaxia, nunca é exagero salientar a importância da educação dos doentes no sentido de saberem como e quando utilizar o kit de adrenalina auto injetável, bem como veicular a noção da ineficácia da pré-medicação com antihistamínicos, os quais podem até mascarar sintomas prodrômicos, impedindo os doentes de preverem reações mais graves.
\end{abstract}

Palavras-chave: Anafilaxia induzida pelo exercício físico; Crise anafilática iminente; Pacientes expostos a crise anafilática iminente.

\section{Introdução}

A anafilaxia é uma condição aguda, rápida, progressiva, geralmente apresentando etiologia alergoimunológica, sendo potencialmente fatal. Acomete qualquer faixa etária e requer pronta atenção e terapia agressiva visando o imediato retorno à normalidade, sendo 
as causas estabelecidas ou não. A anafilaxia induzida por exercício pode ou não ter dependência alimentar, o que faz da anafilaxia induzida por exercício dependente de alimentos (AIEDA) um tipo particular de anafilaxia de exercício (AE), caracterizada pelo aparecimento de uma reação alérgica sistêmica desencadeada pelo exercício (durante ou após), apenas quando este é efetuado nas primeiras 2 horas após a ingestão alimentar (Geller,2013; Caiado, 2007).

Derivada do inglês, a sigla WDEIA (Wheat-Dependent Exercise-Induced Anaphylaxis), na tradução brasileira, AIEDA, caracteriza-se como uma forma especial de anafilaxia induzida pelo exercício físico dependente da ingestão de alimento, que nesse caso é o trigo. Assim o paciente exibe sintomas alérgicos após uma combinação de ingestão de trigo associada a cofatores como o exercício (Scherf et al., 2015; Geller, 2013; Ahanchian at al, 2013). A frequência de WDEIA em pacientes japoneses com AIEDA era de $56 \%$, mas os dados sobre a prevalência de WDEIA em diferentes países são escassos (Scherf et al, 2015; \& Cze-Ja \& John, 2015).

Vários alimentos podem causar esse tipo de reação autoimune, incluindo crustáceos, trigo e derivados, vegetais, frutas, nozes, ovos, cogumelos, milho, alho, carne de porco e carne bovina ou arroz. Desses, os crustáceos, como por exemplo o camarão, e o trigo, são os alimentos mais relacionados com esse tipo de anafilaxia. Entretanto, o trigo parece estar mais associado à AIEDA, visto que houve considerável aumento nos relatos de estudos sobre a associação do trigo com AIE nos últimos anos, principalmente no Japão e em países europeus (Sarinho \& Lins,2017).

Os estudos sobre este tipo de alergia ainda são escassos, principalmente a cerca de dados epidemiológicos, os quais apresentam algumas divergências. Para Scherf et al., (2015) a anafilaxia induzida pelo exercício (AIE) está associada a 5\% à $15 \%$ de todos os casos de anafilaxia e pelo menos $33 \%$ à $50 \%$ dos pacientes com AIE sofrem de AIEDA em estudos norte-americanos. Geller (2013), destaca que no Brasil, a prevalência da anafilaxia induzida por exercícios é de $2,36 \%$ à 5,0\% em todos os casos de anafilaxia.

Dada a raridade desta patologia no contexto clínico, poucos estudos epidemiológicos têm sido efetuados para estimar a sua prevalência. Dados disponíveis em dois estudos japoneses, que envolveram, respectivamente, 76229 adolescentes em um e 11 642 crianças e adolescentes no outro, com prevalência estimada de $0,017 \%$ no primeiro e, no segundo estudo, de $0,06 \%$ em crianças em idade escolar e de 0,21\% em adolescentes. De uma forma geral, os indivíduos são habitualmente adultos jovens (idade média de 37,5 
anos), com marcada predominância do sexo feminino, com razão aproximadamente de 2,5:1 em relação ao sexo masculino (Guanilo, 2011; Roberts \& Bem-Shoshan, 2015).

Por inúmeras vezes a anafilaxia induzida pelo exercício dependente de alimento é subdiagnosticada pelo médico e acaba sendo relatada como uma anafilaxia idiopática. Desse modo, o paciente não recebe as devidas orientações para o correto manejo de sua doença. Sendo assim, não saberá como agir, seja para evitá-la ou para intervir em uma possível crise, logo estará potencialmente sujeito à situações de risco de vida.

Nesse sentido, faz-se a seguinte pergunta norteadora de pesquisa utilizando o acrônimo PICO: P(população):pacientes portadores de AIEDA, principalmente crianças, adolescentes e adultos jovens; I (intervenção): medidas educacionais destinadas a assegurar o correto manejo em uma crise anafilática iminente; $\mathbf{C}$ (contexto): pacientes com AIEDA expostos a uma crise anafilática iminente; e $\mathbf{O}$ (desfecho): melhorar o prognóstico desses pacientes, reduzindo o número de eventos fatais.

Desse modo a pergunta de pesquisa ficou assim delineada: "Como o paciente com anafilaxia induzida pelo exercício dependente de alimento (AIEDA) deve agir diante de uma provável crise, particularmente, na redução do número de eventos fatais?"

\section{Método}

A revisão sistemática da literatura busca superar possíveis vieses em todas as etapas, seguindo um método rigoroso de seleção de pesquisas; avaliação da relevância e da veracidade das pesquisas encontradas; além de coleta, síntese e interpretação dos dados provenientes das pesquisas (Guanilo, 2011).

Essa revisão foi baseada no documento intitulado: Diretrizes metodológicas, elaboração de revisões sistemáticas e metanálise de estudos observacionais comparativos sobre fatores de risco e prognóstico do Ministério da Saúde (BRASIL, 2014). Nesse sentido, seguiu sete passos conforme estabelecido na figura 1 , a seguir. 
Figura 1: Passos seguidos nessa revisão sistemática da literatura.

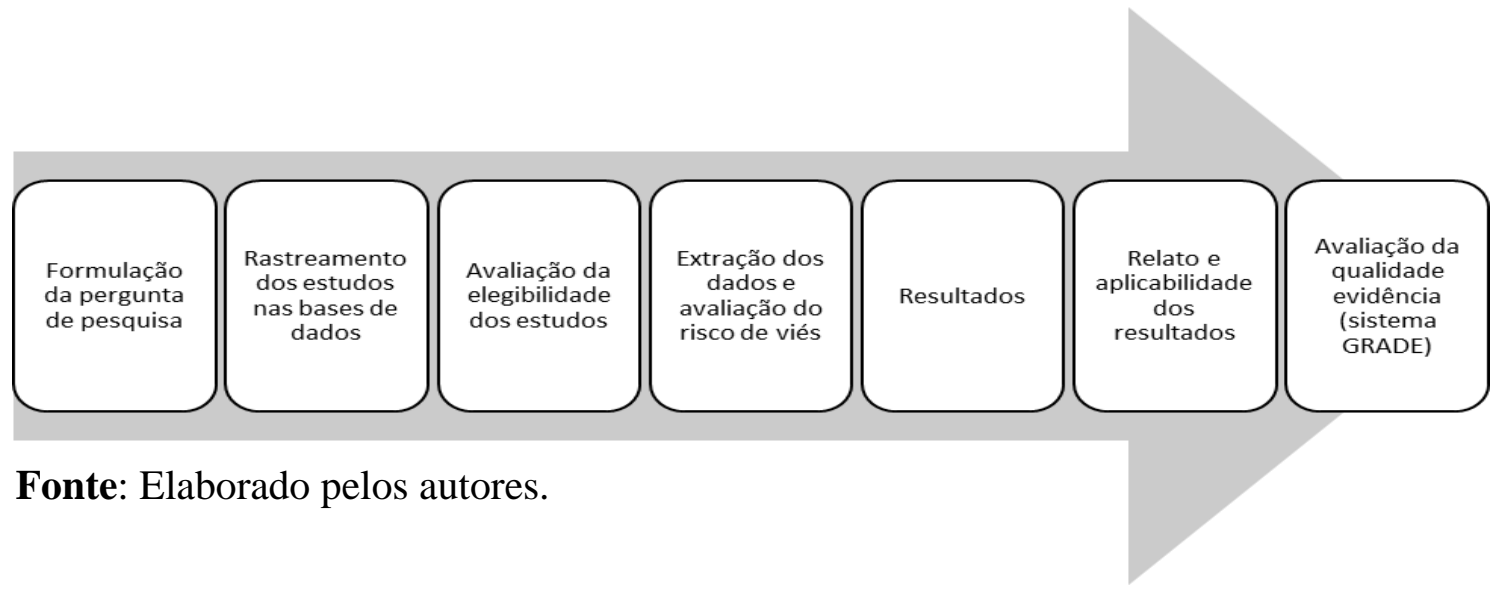

\section{Busca de Potenciais Estudos Elegíveis}

\section{Fontes de Informação (Bases de Dados)}

O processo de busca dos artigos foi estruturado para resgatar o maior número de estudos primários possíveis, objetivando um levantamento amplo da literatura médica sobre o tema, através do Protocolo PRISMA - Principais itens a serem relatados em Revisões sistemáticas e metanálises (http://www.prisma-statement.org/).

Para isso, as seguintes bases foram elencadas devido a sua relevância, gratuidade de acesso e ao elevado número de estudos presentes. A saber: Sistema Online de Busca e Análise de Literatura Médica (MEDLINE), Scientific Eletronic Library Online (SCIELO).

\section{Estratégia de Busca}

As bases de dados possuem descritores de assuntos específicos para cada base, sendo que em algumas bases as buscas são realizadas apenas por palavras-chaves. Dessa maneira, para a localização dos estudos foram selecionados os seguintes descritores controlados: "Anafilaxia induzida pelo exercício físico" (Wheat-Dependent Exercise- 
Induced Anaphylaxis), "Crise anafilática iminente" (Impending anaphylactic crisis), "pacientes expostos a crise anafilática iminente" (patients exposed to impending anaphylactic crisis); estando todos presentes no Medical Subject Headings (MeSH), que é

o dicionário de sinônimos de vocabulário controlado usado para indexar artigos para o PubMed, e nos Descritores em Ciências da Saúde (DeCS), vocabulário trilíngue que serve como linguagem única para indexação de artigos na Biblioteca Virtual em Saúde (Figura 2).

A pesquisa nas bases foi realizada por meio da seguinte equação de busca: "Anafilaxia induzida pelo exercício físico" AND "pacientes expostos a crise anafilática iminente "OR "Crise anafilática iminente".

\section{Extração dos Dados}

A extração dos dados de cada estudo para análise e interpretação foi norteada pelos seguintes pontos: identificação, instituição sede do estudo, instituições envolvidas no estudo, área de publicação, características metodológicas, implicações da pesquisa e qualidade da evidência (Sistema GRADE).

Foram incluídos na amostra 10 estudos (n), que possuíam pacientes com histórico de anafilaxia induzida por exercício, anafilaxia por exercício dependente de alimento, anafilaxia induzida dependente de medicamentos. 


\section{Resultados}

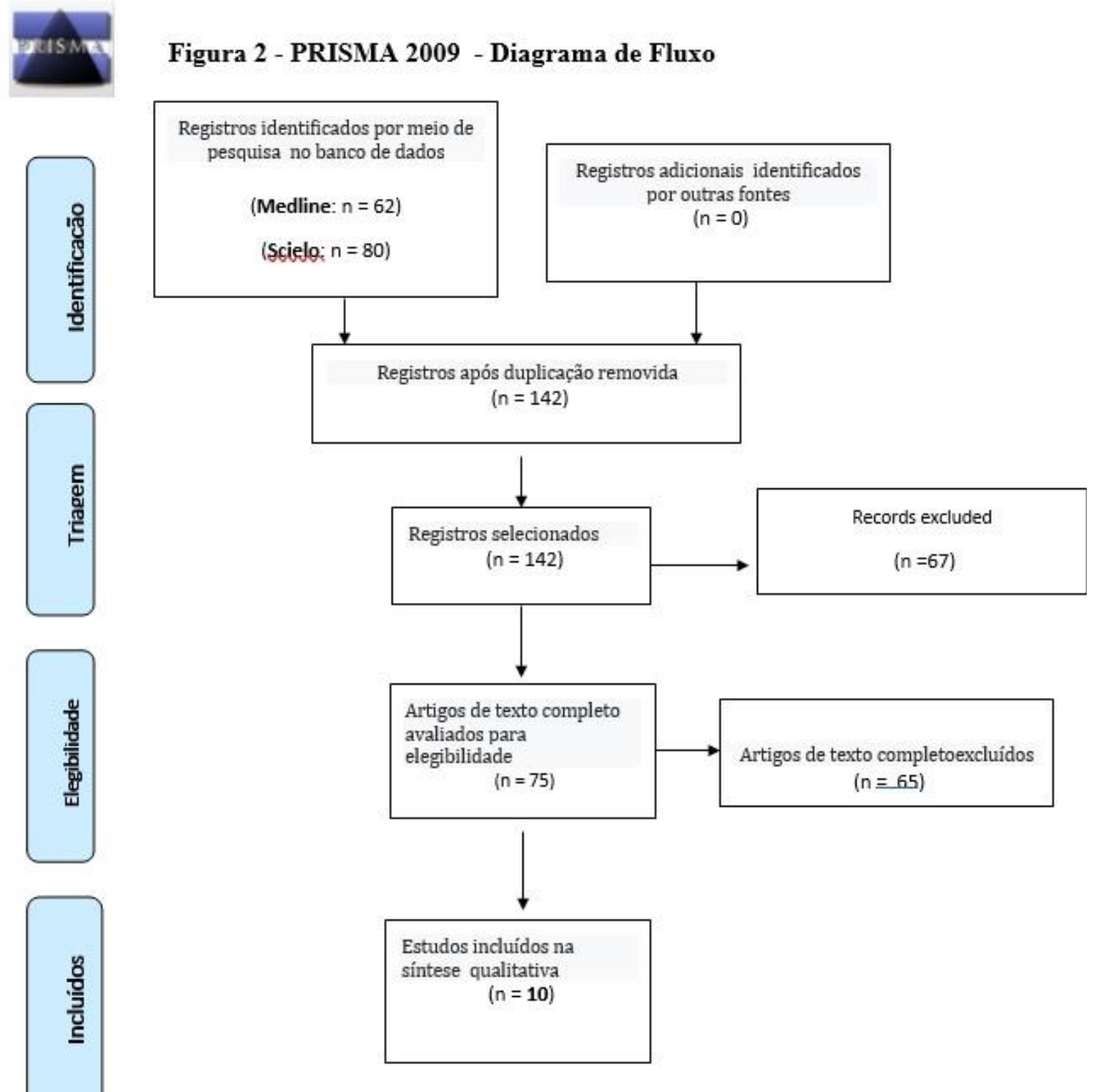

Fonte: Representação esquemática dos estudos incluídos na revisão sistemática usando uma lista de verificação e diagrama de fluxo do Protocolo PRISMA. 
Tabela 1 - Matriz representativa da síntese dos dados do presente estudo.

\begin{tabular}{|c|c|c|c|}
\hline Autor(es) & Journal & Amostra & $\begin{array}{c}\text { Principais } \\
\text { Achados }\end{array}$ \\
\hline Scherf, K. A. et al., 2015. & $\begin{array}{l}\text { Clinical \& } \\
\text { Experimental } \\
\text { Allergy }\end{array}$ & $\begin{array}{l}\text { Em alguns pacientes, } \\
\text { o AIEDA foi discutido } \\
\text { como causado pela } \\
\text { sensibilização } \\
\text { epicutânea com glúten } \\
\text { de trigo hidrolisado } \\
\text { incluído em } \\
\text { cosméticos. O } \\
\text { diagnóstico foi feito } \\
\text { com base na história } \\
\text { do paciente em } \\
\text { combinação com teste } \\
\text { cutâneo de alergia, } \\
\text { determinação de } \\
\text { anticorpos séricos IgE } \\
\text { específicos do trigo, } \\
\text { teste de ativação de } \\
\text { basófilos, teste de } \\
\text { liberação de histamina } \\
\text { e / ou teste de desafio } \\
\text { ao exercício. }\end{array}$ & $\begin{array}{l}\text { A anafilaxia induzida } \\
\text { pelo exercício dependente } \\
\text { de trigo (AIEDA) é uma } \\
\text { alergia alimentar rara, } \\
\text { mas potencialmente } \\
\text { grave, que ocorre } \\
\text { exclusivamente quando a } \\
\text { ingestão de trigo é } \\
\text { acompanhada por } \\
\text { cofatores crescentes. É } \\
\text { clinicamente } \\
\text { caracterizado por reações } \\
\text { anafiláticas que variam } \\
\text { de urticária e angioedema } \\
\text { a dispneia, hipotensão, } \\
\text { colapso e choque }\end{array}$ \\
\hline Caiado, Joana et al., 2007. & $\begin{array}{l}\text { Revista } \\
\text { Portuguesa } \\
\text { Imuno- } \\
\text { alergologia }\end{array}$ & 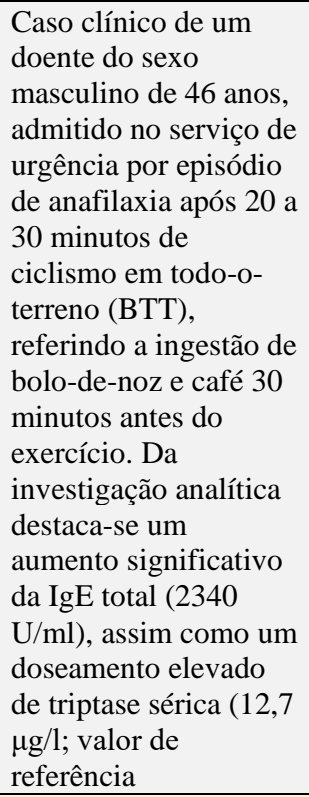 & $\begin{array}{l}\text { O diagnóstico de AIEDA } \\
\text { baseia-se em critérios } \\
\text { clínicos, e requer a } \\
\text { combinação de ingestão } \\
\text { alimentar e exercício para } \\
\text { o aparecimento de } \\
\text { sintomas sistémicos. No } \\
\text { caso descrito, trata-se de } \\
\text { AIEDA específica para a } \\
\text { noz. Apesar da IgE } \\
\text { específica negativa, a } \\
\text { sensibilização à noz é } \\
\text { comprovada pelos testes } \\
\text { cutâneos positivos com } \\
\text { este alimento. As } \\
\text { cicloergometrias } \\
\text { negativas permitiram } \\
\text { excluir a anafilaxia de } \\
\text { exercício e a provocação } \\
\text { com noz excluíu a alergia } \\
\text { alimentar }\end{array}$ \\
\hline Geller, Mario. 2013. & $\begin{array}{l}\text { Brazilian } \\
\text { Journal of } \\
\text { Allergy and } \\
\text { Immunologia }\end{array}$ & $\begin{array}{l}\text { Revisão da literature } \\
\text { que descreve os } \\
\text { aspectos clínicos, } \\
\text { diagósticos e manejo } \\
\text { da anafilaxia induzida } \\
\text { por exercício, } \\
\text { anafilaxia por } \\
\text { exercicio dependente } \\
\text { de alimento, anafilaxia } \\
\text { dependente de } \\
\text { medicamentos e } \\
\text { anafilaxia idiopática.. }\end{array}$ & $\begin{array}{l}\mathrm{Na} \text { anafilaxia idiopática, } \\
\text { degranulação de } \\
\text { mastóscitos foi } \\
\text { documentada, com } \\
\text { liberação de histamina e } \\
\text { triptase. Anafilaxia } \\
\text { induzida por exercício e } \\
\text { anafilaxia induzida por } \\
\text { exercício dependente de } \\
\text { alimentos são condições } \\
\text { raras, mas potencialmente } \\
\text { ameceadoras à vida }\end{array}$ \\
\hline $\begin{array}{l}\text { Sarinho, Emanuel; LINS, } \\
\text { Maria das Graças Moura. } 2017 .\end{array}$ & $\begin{array}{l}\text { Jornal de } \\
\text { Pediatria }\end{array}$ & $\begin{array}{l}\text { Gerenciamento } \\
\text { diagnóstico e } \\
\text { terapêutico de formas } \\
\text { graves de alergia } \\
\text { alimentar, pesquisados } \\
\text { no banco de dados }\end{array}$ & $\begin{array}{l}\text { Alergia alimentar grave } \\
\text { pode ocorrer na forma de } \\
\text { anafilaxia e síndrome de } \\
\text { enterocolite induzida por } \\
\text { proteínas alimentares, } \\
\text { cada vez mais observada }\end{array}$ \\
\hline
\end{tabular}




\begin{tabular}{|c|c|c|c|}
\hline & & $\begin{array}{l}\text { Medline usando os } \\
\text { termos "alergia } \\
\text { alimentar grave", } \\
\text { "anafilaxia e alergia } \\
\text { alimentar", "urticária } \\
\text { generalizada e alergia } \\
\text { alimentar" e } \\
\text { "enterocolite induzida } \\
\text { por proteínas } \\
\text { alimentares"síndrome" } \\
\text { nos últimos dez anos, } \\
\text { pesquisando nos } \\
\text { campos de título, } \\
\text { resumo ou palavra- } \\
\text { chave. }\end{array}$ & $\begin{array}{l}\text { na sala de emergência } \\
\text { pediátrica; portanto, o } \\
\text { pediatra deve estar alerta } \\
\text { para poder fornecer o } \\
\text { diagnóstico e tratamento } \\
\text { imediatos. }\end{array}$ \\
\hline Ahanchian, H. et al., 2013. & $\begin{array}{l}\text { Iran Journal } \\
\text { Allergy } \\
\text { Asthma } \\
\text { Immunololy }\end{array}$ & $\begin{array}{l}\text { Neste estudo, uma } \\
\text { mulher de } 32 \text { anos } \\
\text { visitou a clínica de } \\
\text { alergias com uma } \\
\text { história de vários } \\
\text { episódios de colmeias } \\
\text { desde } 11 \text { anos atrás e } \\
\text { três ataques de } \\
\text { anafilaxia com risco } \\
\text { de vida nos últimos } 6 \\
\text { meses. O início da } \\
\text { maioria desses ataques } \\
\text { ocorreu devido à } \\
\text { atividade física após o } \\
\text { café da manhã.. }\end{array}$ & $\begin{array}{l}\text { Nos testes de picada na } \\
\text { pele para alérgenos } \\
\text { alimentares comuns, ela } \\
\text { mostrou uma pápula de } 6 \\
\text { milímetros com } \\
\text { alargamento de } 14 \mathrm{~mm} \text { no } \\
\text { extrato de trigo. O } \\
\text { restante dos alérgenos foi } \\
\text { negativo. A paciente foi } \\
\text { diagnosticada como } \\
\text { induzida pelo exercício } \\
\text { dependente do trigo, e } \\
\text { todos os alimentos que } \\
\text { continham trigo foram } \\
\text { omitidos de sua dieta }\end{array}$ \\
\hline $\begin{array}{l}\text { Wong, GK; } \\
\text { Krishna, MT; } \\
\text { Wong and Mamidipudi T. } \\
\text { Krishna, } \\
2013 \text {. }\end{array}$ & $\begin{array}{l}\text { Current } \\
\text { Allergy and } \\
\text { Asthma } \\
\text { Reports }\end{array}$ & $\begin{array}{l}\text { Esta revisão faz } \\
\text { comparações entre } \\
\text { anafilaxia induzida } \\
\text { por exercício } \\
\text { dependente de trigo } \\
\text { (WDEIA) e outra } \\
\text { anafilaxia induzida } \\
\text { por exercício } \\
\text { dependente de } \\
\text { alimento (FDEIAs) e } \\
\text { discute a importância } \\
\text { de co-fatores em sua } \\
\text { fisiopatologia }\end{array}$ & $\begin{array}{l}\text { Em uma escala maior, a } \\
\text { FDEIA (Anafilaxias } \\
\text { induzidas pelo exercício, } \\
\text { dependentes de } \\
\text { alimentos) representa um } \\
\text { elo crucial entre reações } \\
\text { mediadas por IgE e } \\
\text { reações anafilactóides e } \\
\text { fornece evidências de } \\
\text { suporte para o conceito } \\
\text { de 'anafilaxia de } \\
\text { somatória' e a } \\
\text { necessidade de superar o } \\
\text { 'limiar alérgico' }\end{array}$ \\
\hline $\begin{array}{l}\text { Roberts, Hannah; } \\
\text { Ben-Shoshan, Moshe, } \\
2015 .\end{array}$ & $\begin{array}{l}\text { Journal of } \\
\text { Medical } \\
\text { Case Reports }\end{array}$ & $\begin{array}{l}\text { Caso de anafilaxia } \\
\text { induzida por exercício, } \\
\text { dependente de comida, } \\
\text { em uma menina } \\
\text { branca de } 17 \text { anos de } \\
\text { idade devido ao grão } \\
\text { de bico. Ela tinha } \\
\text { histórico de anafilaxia } \\
\text { após comer bolachas e } \\
\text { hummus antes de se } \\
\text { exercitar. O teste de } \\
\text { picada na pele e o } \\
\text { nível de } \\
\text { imunoglobulina E } \\
\text { específico do soro } \\
\text { confirmaram o grão de } \\
\text { bico como alérgeno } \\
\text { causador. }\end{array}$ & $\begin{array}{l}\text { Embora exista anafilaxia } \\
\text { induzida por exercício, } \\
\text { dependente de comida e } \\
\text { rara, e fazer um } \\
\text { diagnóstico possa levar a } \\
\text { estratégias preventivas } \\
\text { que salvam vidas. Os } \\
\text { vegetais não são um } \\
\text { alimento comum } \\
\text { associado à anafilaxia } \\
\text { induzida pelo exercício, } \\
\text { dependente de alimentos; } \\
\text { isso aumentará nossa } \\
\text { base de conhecimento } \\
\text { atual no campo da } \\
\text { alergia. }\end{array}$ \\
\hline $\begin{array}{l}\text { Cze-Ja Tam; } \\
\text { JOHN, Rita Marie, } \\
2017 .\end{array}$ & $\begin{array}{l}\text { The Journal } \\
\text { for Nurse } \\
\text { Practitioners }\end{array}$ & $\begin{array}{l}\text { Revisar as } \\
\text { características da } \\
\text { FDEIA, as } \\
\text { ferramentas de }\end{array}$ & $\begin{array}{l}\text { Um diagnóstico adequado } \\
\text { da FDEIA ajudará a } \\
\text { evitar limitações } \\
\text { desnecessárias de }\end{array}$ \\
\hline
\end{tabular}




\begin{tabular}{|c|c|c|c|}
\hline & & $\begin{array}{l}\text { diagnóstico, o } \\
\text { gerenciamento e o } \\
\text { papel do enfermeiro } \\
\text { no diagnóstico e } \\
\text { atendimento de } \\
\text { crianças ou } \\
\text { adolescentes com } \\
\text { suspeita de FDEIA. }\end{array}$ & $\begin{array}{l}\text { alimentos e exercícios e } \\
\text { proporcionará ao paciente } \\
\text { com FDEIA um estilo de } \\
\text { vida adaptável }\end{array}$ \\
\hline $\begin{array}{l}\text { Brockow, K. et al., } \\
2015 .\end{array}$ & $\begin{array}{l}\text { Journal } \\
\text { Allergy } \\
\text { Clinical } \\
\text { Immunology }\end{array}$ & $\begin{array}{l}\text { Procuramos confirmar } \\
\text { o diagnóstico de } \\
\text { WDEIA usando o } \\
\text { desafio de farinha de } \\
\text { glúten oral e cofatores, } \\
\text { para determinar a } \\
\text { quantidade de glúten } \\
\text { necessária para } \\
\text { desencadear sintomas } \\
\text { e correlacionar esses } \\
\text { resultados com os } \\
\text { níveis plasmáticos de } \\
\text { gliadina, } \\
\text { permeabilidade } \\
\text { gastrointestinal e } \\
\text { parâmetros } \\
\text { alergológicos. }\end{array}$ & $\begin{array}{l}\text { A provocação oral com } \\
\text { glúten isolado ou } \\
\text { juntamente com AAS e } \\
\text { álcool é um teste sensível } \\
\text { e específico para o } \\
\text { diagnóstico de WDEIA. } \\
\text { O exercício não é um } \\
\text { gatilho essencial para o } \\
\text { aparecimento dos } \\
\text { sintomas em pacientes } \\
\text { com WDEIA. }\end{array}$ \\
\hline $\begin{array}{l}\text { Morita, Eishin; } \\
\text { Chinuki,Yuko; } \\
\text { Takahashi, Hitoshi. } \\
2013 \text {. }\end{array}$ & $\begin{array}{l}\text { Journal of } \\
\text { Dermatologi } \\
\text { cal Science }\end{array}$ & $\begin{array}{l}\text { Como os alimentos } \\
\text { causadores e a } \\
\text { condição dos gatilhos } \\
\text { variam entre os } \\
\text { pacientes, o } \\
\text { diagnóstico de FDEIA } \\
\text { nem sempre é fácil. Os } \\
\text { testes IgE séricos } \\
\text { específicos de } \\
\text { alimentos, } \\
\text { amplamente utilizados } \\
\text { no diagnóstico de } \\
\text { FDEIA, têm } \\
\text { sensibilidade bastante } \\
\text { baixa, porque os testes } \\
\text { utilizam } \\
\text { principalmente } \\
\text { extratos brutos de } \\
\text { alimentos. O conceito } \\
\text { de uso de moléculas } \\
\text { definidas de alérgeno } \\
\text { foi proposto como o } \\
\text { termo "diagnóstico } \\
\text { resolvido por } \\
\text { componentes" para o } \\
\text { diagnóstico de alergia } \\
\text { mediada por IgE. }\end{array}$ & $\begin{array}{l}\text { É relatado que o teste de } \\
\text { ativação de basófilos } \\
\text { (BAT) baseado em } \\
\text { expressão de CD203c é } \\
\text { útil na identificação de } \\
\text { pacientes adultos com } \\
\text { WDEIA e na previsão de } \\
\text { alérgenos causadores no } \\
\text { WDEIA, quando } \\
\text { combinado com } \\
\text { alérgenos apropriados. A } \\
\text { detecção dos níveis } \\
\text { séricos de alérgenos } \\
\text { possivelmente fornece } \\
\text { informações úteis se os } \\
\text { testes de desafio } \\
\text { alimentar foram } \\
\text { realizados com força } \\
\text { suficiente. }\end{array}$ \\
\hline
\end{tabular}

Fonte: Dados da Pesquisa, 2019.

\section{Discussão}

A anafilaxia é uma reação de hipersensibilidade generalizada ou sistêmica, potencialmente fatal, envolvendo vários órgãos e sistemas, particularmente a pele, o trato 
respiratório, o trato gastrointestinal e o sistema cardiovascular (Scherf et al., 2015). Acredita-se que a anafilaxia induzida pelo exercício (AIE) esteja associada a $5-15 \%$ de todos os casos de anafilaxia. Os dados de prevalência da AIEDA são limitados e não relatados com exatidão. A maioria dos casos relatados tem sido relacionada ao trigo. A prevalência relatada variou de $0,001 \%$ a $0,017 \%$ em todo o mundo para todos os alimentos. A AIEDA continua sendo subnotificada, com apenas 700 casos estudados e reconhecidos no mundo, estudos mostram que $5 \%$ a $15 \%$ dos casos de anafilaxia são causados por AIE, e dos pacientes com AIE, 33\% a 50\% apresentam AIEDA. A maioria dos estudos afirma que a AIEDA parece ser duas vezes mais comum em homens que em mulheres com a maior prevalência entre a adolescência e 35 anos; no entanto, ocorre em menor proporção em crianças, provavelmente devido a casos não relatados (Cze-Ja \& John, 2017).

Muito se tem especulado sobre a fisiopatologia desta síndrome, embora estejam já descritos alguns mecanismos potencialmente responsáveis. $\mathrm{Na}$ maioria dos doentes, quando há uma reação IgE mediada, verifica-se que o exercício diminui o limiar para a degranulação dos mastócitos, dando origem aos sintomas, mesmo quando a ingestão isolada é tolerada. Está demonstrado que o exercício aumenta a permeabilidade intestinal, sendo assim responsável pelo aumento da absorção intestinal das fracções alergênicas dos alimentos (Wong \& Krishma, 2013; Geller, 2013).

Algumas vezes, o quadro de alergia alimentares graves se desenvolve durante o exercício em associação com um alimento especifico (até quatro horas após a ingestão), caracteriza a anafilaxia induzida pelo exercício dependente de alimento. $\mathrm{O}$ exercício pode promover uma maior absorção do alérgeno inadequadamente processado e/ou promovera degranulação de basófilos e mastócitos sensibilizados ou ainda promover um excesso de síntese de metabólitos do ácido araquidônico. Nesse caso, a anafilaxia é resultante da associação do alimento e do exercício, enquanto o alimento e o exercício, quando acontecem isolados, são bem tolerados. O trigo é o mais comum, mas outros grãos, nozes e outros alimentos também têm sido incriminados (Sarinho \& Lins, 2017).

Para evitar mais episódios de WDEIA, a educação dos pacientes é crucial e eles devem receber um plano de manejo de anafilaxia por escrito, bem como orientação nutricional. As medidas terapêuticas além das preventivas, já anteriormente mencionadas, incluem: a disponibilidade de epinefrina autoinjetora, a realização dos exercícios sempre acompanhados por indivíduos que também saibam reconhecer a anafilaxia e dominem a técnica do emprego intramuscular na face ântero-lateral da coxa da epinefrina autoinjetora, 
ter sempre um celular disponível, estar próximo a centros de tratamento médico emergencial, não praticar exercícios em trilhas afastadas, usar um alerta médico do tipo bracelete ou cordão, identificando o problema, e os telefones para contato imediato de urgência. (Geller 2013; Scherf et al., 2015).

Os estudos no Japão também confirmam que os alimentos causadores mais comuns são produtos de trigo e crustáceos. O trigo como protagonista na anafilaxia induzida pelo exercício dependente do trigo, foi o alimento mais estudado na AIEDA ao longo das últimas décadas. Devido à sua popularidade, tornou-se um modelo clínico perfeito para AIEDA, mas não é de forma alguma uma condição única. Assim, ele ajudou a entender a apresentação clínica da AIEDA, o qual representa um elo crucial entre as reações anafiláticas e mediadas por IgE, e fornece evidência de apoio para o conceito de "anafilaxia por somação" e a necessidade de superar o limiar de alérgeno. Atualmente, não existem métodos para prever o episódio inicial, assim a ênfase está na prevenção do segundo ou do último episódio assim como as orientações do estilo de vida para prevenção da anafilaxia instrui a não consumir qualquer alimento causativo dentro de $2 \mathrm{~h}$ antes do exercício. No entanto, é preciso cautela para evitar a eliminação completa dos alimentos causadores e a restrição do exercício físico. (Wong \& Krishna 2013; Brockow et al., 2015; Morita, Chinuki \& Takahashi, 2013).

\section{Conclusões}

O diagnóstico e o manejo apropriados pelo profissional ajudará a evitar limitações desnecessárias na dieta e no exercício do paciente. Os pacientes devem usar pulseira médica, bem como canetas de adrenalina e anti-histamínicos auto injetáveis prescritos em caso de exposição acidental durante exercícios leves ou vigorosos. Ao se exercitar, o indivíduo com AIEDA deve ter um amigo e/ou familiar que entenda a AIEDA e como administrar epinefrina em caso de emergência (Cze-Ja \& John, 2017; Geller 2013).

É importante que a família, os amigos e os líderes comunitários reconheçam cada vez mais os sinais precoces e que saibam manejar a anafilaxia com adrenalina auto injetável ou mesmo providenciar e treinar o uso da medicação montada em seringa quando essa apresentação não estiver disponível. Perante qualquer situação de anafilaxia, nunca é exagero salientar a importância da educação dos doentes no sentido de saberem como e quando utilizar o kit de adrenalina auto injetável, bem como veicular a noção da ineficácia 
da pré-medicação com anti-histamínicos, os quais podem até mascarar sintomas prodrômicos, impedindo os doentes de preverem reações mais graves. Além disso, a sua utilização nunca obvia uma observação subsequente, tão rápida quanto possível, em serviço de urgência. (Sarinho \& Lins, 2017).

As evidências apontam que AIEDA é um tipo de anafilaxia rara e, por isso, deve ser diagnosticada de forma rápida para evitar desfechos fatais. Dessa forma, é essencial a elaboração de uma história clínica detalhada com a finalidade de identificar a associação entre a ingestão alimentar e o início dos sintomas, quer seja durante ou após o exercício.

É condição sine qua non o diagnóstico predominantemente clínico, com base na anamnese e exame físico, o que irá ser um contributo importante para alguns exames laboratoriais que se façam necessários para confirmação e/ou a determinação do IgE específico e testes de provocação com alimento e exercício.

\section{Referências}

Ahanchian, H. et al. (2013). Food-Dependent Exercise-Induced Anaphylaxis due to Wheat in a Young Woman. Iran Journal Allergy Asthma Immunololy, v.12, n.1, p.93-95. Disponível em: https://www.ncbi.nlm.nih.gov/pubmed/23454785

Brasil. Ministério da Saúde. Secretaria de Ciência, Tecnologia e Insumos Estratégicos. Departamento de Ciência e Tecnologia (2014). Diretrizes metodológicas, elaboração de revisões sistemáticas e metanálise de estudos observacionais comparativos sobre fatores de risco e prognóstico.Brasília : Ministério da Saúde. Disponível em: file:///C:/Users/HOME/Downloads/diretriz_metodologia_05\%20(1).pdf

Brockow, K. et al. (2015). Using a gluten oral food challenge protocol to improve diagnosis of wheat-dependent exercise-induced anaphylaxis. Journal Allergy Clinical Immunology, v.135, n.4, p. 977-984. Disponível em: https://www.ncbi.nlm.nih.gov/pubmed/25269870

Caiado, Joana et al. (2007). Food-dependent exercise-induced anaphylaxis. Revista Portugiesa Imunoalergologia, v.15, n.2, p.179-187. Disponívem em: https://www.spaic.pt/client_files/rpia_artigos/anafilaxia-induzida-por-exerciciodependente-de-alimentos-\%E2\%80\%93-caso-clinico.pdf

Cze-Ja Tam; John, Rita Marie (2017). Food-Dependent Exercise-Induced Anaphylaxis: A Review. The Journal for Nurse Practitioners, v.13, n.5, p. 314-321. Disponível em: https://www.npjournal.org/article/S1555-4155(17)30025-9/fulltext 
Geller, Mario (2013). The multiple faces of anaphylaxis: exercise-induced anaphylaxis and idiopathic anaphylaxis. Brazilian Journal of Allergy and Immunologia, v.1, n.1, p. 8-13. Disponíve em: http://aaai-asbai.org.br/detalhe_artigo.asp?id=8

Guanilo, Mônica Cecilia De-la-Torre-Ugarte; Takahashi, Renata Ferreira; Bertolozzi, Maria Rita (2011). Revisão sistemática: noções gerais. Revista da Escola de Enfermagem da USP, v.45, n.5, p. 1260-1266. Disponível em: http://www.scielo.br/scielo.php?script=sci_arttext\&pid=S0080-62342011000500033

Morita, Eishin; Chinuki,Yuko; Takahashi, Hitoshi. (2013). Recent advances of in vitro tests for the diagnosis of food-dependent exercise-induced anaphylaxis. Journal of Dermatological Science, v.71, n.1, p. 155-159. Disponível em: https://www.ncbi.nlm.nih.gov/pubmed/23669019

Roberts, Hannah; BEN-SHOSHAN, Moshe (2015). Food-dependent exercise-induced anaphylaxis to chickpea in a 17-year-old female: a case report. Journal of Medical Case Reports, $\quad$ v.9, n.186, p.1-4.. https://www.ncbi.nlm.nih.gov/pubmed/26334308

Sarinho, Emanuel; LINS, Maria das Graças Moura (2017). Severe forms of food allergy. Jornal de Pediatria, v.93, n.1, p. 53-59. Disponível em: http://jped.elsevier.es/pt-severeforms-food-allergy-articulo-S2255553617301465

Scherf, K. A. et al. (2015). Wheat-dependent exercise-induced anaphylaxis. Clinical \& Experimental Allergy, v.46, p. 10-20. Disponível em: https://www.ncbi.nlm.nih.gov/pubmed/26381478;

Wong, GK; Krishna, MT. (2013). Food-Dependent Exercise-Induced Anaphylaxis: Is Wheat Unique? Current Allergy and Asthma Reports, v.13, p. 639-644. Disponível em: https://link.springer.com/article/10.1007\%2Fs11882-013-0388-2.

\section{How to cite this article (APA format):}

Santos Filho, Antonio Adriano Gomes dos; Rolim Neto, Modesto Leite (2019). FoodDependent Exercise Anaphilaxy: A Systematic Review of Literature. Am. In. Mult. J., October. (7) 4, 148-161.

Received: $11 / 25 / 2019$

Accepted: 11/30/2019 